\title{
A computationally efficient moment-preserving Monte Carlo proton transport method in Geant4
}

\author{
D. A. Dixon ${ }^{1}$, A. K. Prinja ${ }^{2}$, A. P. McCartney ${ }^{1}$, and H. G. Hughes ${ }^{1}$ \\ ${ }^{1}$ Los Alamos National Laboratory, Los Alamos, NM 87545 \\ ${ }^{2}$ University of New Mexico, Dept. of Nuclear Engineering, Albuquerque, NM 87131
}

\begin{abstract}
The moment-preserving method, demonstrated as a viable alternative to condensed history for electrons, is extended to protons. Given the generality and the flexibility of the method, a discrete Coulomb scattering and discrete impactionization differential cross-section library for protons was readily developed and existing Geant 4 electron discrete process and model classes were extended to make use of the new proton library. It is shown that levels of efficiency and accuracy similar to those demonstrated for electrons are obtainable for protons in straight-ahead, energy-loss problems. However, in problems with deflection, agreement is strongly dependent on the proton energy. That is, good agreement was observed in the few $\mathrm{MeV}$ range, while unsatisfactory agreement was observed in problems with proton energies above $100-\mathrm{MeV}$.
\end{abstract}

KEYWORDS: MCNP6, Monte Carlo, electron-photon transport, multiple-scattering, validation

\section{Introduction}

The interaction physics for charged particles such as electrons, protons, and heavy ions is characterized by extremely short distances to collisions typically followed by an infinitesimally small change in the particle direction and energy. ${ }^{(\mathbf{1})}$ The associated single-scatter differential cross sections (DCS) are nearly singular about zero energy transfers and angular deflections rendering single-scatter Monte Carlo charged particle transport (CPT) simulations computationally impractical without significant computing resources. To overcome the computational burden, step-based Monte Carlo methods ${ }^{(2-5)}$ are typically employed. These methods utilize infinite medium multiple scattering $^{(\mathbf{6})}$ and multiple excitation/impact-ionization energy-loss theories ${ }^{(7)}$ condensing large numbers of collisions into individual collisions over a fixed spatial step. However, these schemes are known to suffer (with varying severity) from the inconsistency between the application of deterministic stepping to an inherently stochastic process. As a result, some step-based methods require ad hoc schemes for mitigating the impact of step-size dependencies and boundary crossing approximations.

In this paper, we extend the recently developed momentpreserving (MP) method demonstrated as a viable alternative to the condensed history method for electron transport, ${ }^{(\mathbf{8})}$ to protons. Motivated by Lewis theory, analog DCSs are replaced in this approach by approximate DCSs that preserve a finite number of angular deflection and energy-loss moments of the analog DCS while approximating all remaining moments in terms of the exact lower order moments. This has been shown to yield longer mean free paths and less peaked cross sections, ${ }^{(\mathbf{8})}$ which promotes the use of single-scatter Monte Carlo simulation. Moreover, accuracy scales with the number of moments preserved, while efficiency is achieved in most ap- plications through the need to preserve only a limited number of moments. ${ }^{(\mathbf{8})}$ Finally, the algorithms associated with the MP method are independent of the form of the underlying DCS, whether a theoretical result or tabulated data (numerical or experimental), and apply generically to all charged particle types. Therefore, significant reduction in code development and maintenance can be realized through use of a generic class that is extensible to a variety of charged particle types.

\section{Method}

Herein, we investigate the extension of the MP method to proton transport for three problems: the infinite medium problem (angular deflection only), the straight-ahead problem (energy-loss only), and a 1-D energy deposition problem (both angular deflection and energy-loss). We begin by introducing the analog cross section models used to generate the numerical benchmarks along with the corresponding DCS moments. We then define the discrete MP cross section models and summarize the procedure for computing discrete points and weights.

\section{Differential cross-section models}

The primary proton collision types explored herein are elastic Coulombic collisions with atoms (with cross sections denoted as $\Sigma_{a}$ ) and impact-ionization collisions with atomic electrons (with cross sections denoted as $\Sigma_{e}$ ). For simplicity, we ignore delta-ray electrons, but accounting for delta-rays can be accommodated in the MP method as previously demonstrated. ${ }^{(\mathbf{8})}$ We begin with a discussion of elastic Coulombic collisions with atoms as described by the Wentzel differential cross section. ${ }^{(9,10)}$

The Wentzel cross section describes collisions with atoms 
where particles are deflected through some angle and impart energy to the atom depending on the angle through which the particle was deflected. For particles whose relativistic mass is not much smaller than the relativistic mass of the target, one must consider the cross section in the center-of-mass (COM) frame. The Wentzel differential cross section (units of $\mathrm{cm}^{2}$ ) for protons in the COM frame is given by:

$$
\Sigma_{a}\left(E_{l a b}^{k i n}, \mu\right)=2 \pi\left(\frac{r_{e} Z_{p} Z_{t} m_{e} c^{2}}{\beta_{r} p_{c o m}}\right)^{2}[1+2 \eta+\mu]^{-2},
$$

where

$$
\eta=Z_{t}^{2 / 3}\left(\frac{m_{e} c^{2} \alpha}{a_{T F} p_{c o m}}\right)^{2}\left[1.13+3.76\left(\frac{Z_{p} Z_{t}}{\beta_{r}}\right)^{2}\right],
$$

and $E_{l a b}^{k i n}$ is the kinetic energy of the projectile in the lab frame, $\mu$ is the cosine of the angle between the initial and final momentum vector of the projectile, $r_{e}$ is the classical electron radius, $Z_{p}$ is the atomic number of the projectile, $Z_{t}$ is the atomic number of the target atom, $m_{e} c^{2}$ is the electron rest mass energy, $\alpha$ is the fine structure constant, $a_{T F}=\frac{1}{2}\left(\frac{3 \pi}{4}\right)^{2 / 3}$ is the ThomasFermi constant, $\beta_{r}$ is the particle velocity per $c$ in an "effective" frame, $p_{\text {com }}$ is the COM momentum of the projectile, and $\eta$ is the screening parameter. Definitions of the above terms can be found in the iliterature..$^{(9,10)}$

Impact-ionization collisions are considered those in which the energy transferred by the projectile to a bound electron exceeds the binding energy of the target atom. A model for this collision type is the relativistic Rutherford $\operatorname{DCS}^{(\mathbf{1})}$ for spin $1 / 2$ particles, or:

$$
\begin{aligned}
& \Sigma_{e}\left(E_{\text {lab }}^{k i n}, Q\right)= N_{e} \frac{2 \pi r_{e}^{2} Z_{p}^{2} Z_{t} m_{e} c^{2}}{\beta_{\text {lab }}^{2} Q^{2}} \\
& {\left[1-\beta_{\text {lab }}^{2} \frac{Q}{Q_{\max }}+\frac{1}{2}\left(\frac{Q}{E_{\text {lab }}^{\text {kin }}+m c^{2}}\right)\right], } \\
& Q_{\text {min }} \leq Q \leq Q_{\text {max }},
\end{aligned}
$$

where $Q_{\text {min }}$ is the mean ionization potential,

$$
Q_{\max }=\left(m_{e} c^{2}\right)^{2} \beta_{l a b}^{2} \gamma_{l a b}^{2},
$$

and $\gamma_{l a b}^{2}=1 /\left(1-\beta_{l a b}^{2}\right)$.

These cross sections will be used to establish the numerical benchmarks from which the accuracy and efficiency of the MP method will be evaluated. Furthermore, the MP DCS's are constructed from the moments of the analog DCS cross sections.

\section{Differential cross section moments}

As described in Dixon et al., ${ }^{(8)}$ differential cross section moments are central to the MP method so we now define the relevant DCS moments and then introduce the MP models used in this study.

Legendre moments of the Wentzel DCS in the COM are given by:

$$
\Sigma_{n, \ell}=2 \pi \int_{-1}^{1} P_{\ell}(\mu) \Sigma_{a}\left(E_{l a b}^{k i n}, \mu\right) d \mu
$$

The moments contain important physical quantities such as the collision mean free path, $\lambda_{a}=1 / \Sigma_{a, 0}$, and the transport mean free path, $\lambda_{t r}=1 /\left[\Sigma_{a, 0}-\Sigma_{n, 1}\right]$ which is related to the mean scattering cosine. In charged particle transport $\lambda_{t r} / \lambda_{a}>>1$, which implies that particles undergo a staggering number of collisions before any significant change in the particle direction is observed. There is a well-known recurrence ${ }^{(2)}$ for computing the moments in Eq. (5); however, we use a Gauss-Kronrod quadrature algorithm to compute the Legendre moments.

Now, we present the energy-loss moments of the relativistic Rutherford DCS given in Eq. (3):

$$
Q_{n}(E)=\int_{Q_{\min }}^{Q_{\max }} Q^{n} \Sigma_{e}(E, Q) d Q, n=0,1, \ldots
$$

Defining $C(E)$ as:

$$
C(E)=\frac{4 \pi r_{e}^{2} Z_{1}^{2} Z_{2} m_{e} c^{2}}{\beta^{2}}
$$

and inserting Eq. (3) into Eq. (6) for $n=0$ gives:

$$
\Sigma_{e, 0}=C(E)\left[\left(\frac{1}{Q_{\min }}-\frac{1}{Q_{\max }}\right)-\frac{\beta^{2}}{Q_{\max }} \ln \left(\frac{Q_{\max }}{Q_{\min }}\right)\right],
$$

for $n=1$ :

$$
\Sigma_{e, 1}=C(E)\left[\ln \left(\frac{Q_{\max }}{Q_{\min }}\right)-\beta^{2}\left(1-\frac{Q_{\min }}{Q_{\max }}\right)\right],
$$

and for for $n>1$ :

$\Sigma_{e, n}=C(E)\left[Q_{\max }^{n-1}\left(1-\beta^{2} \frac{n-1}{n}\right)-Q_{\min }^{n-1}\left(1-\beta^{2} \frac{n-1}{n} \frac{Q_{\min }}{Q_{\max }}\right)\right]$.

We note that at high energies, where $Q_{\max }\left(\beta_{l a b}\right)$ can be significant, the higher moments increase with increasing order index $n$, while at lower energies they decrease with increasing $n$. Thus, while moments higher than two are often neglected when describing thick target energy spectra, they play a significant role in the transport of high energy protons through thin targets.

\section{Discrete moment-preserving models}

The form of the MP DCS used herein is referred to as the discrete model and for Coulomb elastic scattering is defined as:

$$
\tilde{\Sigma}_{a}(E, \mu)=\sum_{\ell=1}^{L} \frac{\alpha_{\ell}(E)}{2 \pi} \delta\left[\mu-\xi_{\ell}(E)\right],
$$

where $\alpha_{\ell}$ and $\xi_{\ell}$ are discrete points and weights respectively, and the total cross section is:

$$
\tilde{\Sigma}_{a, 0}=\sum_{\ell=1}^{L} \alpha_{\ell}(E) .
$$

Similarly, discrete impact-ionization DCS is defined as:

$$
\tilde{\Sigma}_{e}(E, Q)=\sum_{k=1}^{K} \beta_{k}(E) \delta\left[Q-\gamma_{k}(E)\right],
$$


where $\gamma_{k}$ and $\beta_{k}$ are the discrete points and weights respectively, and the total cross section is:

$$
\tilde{\Sigma}_{e, 0}=\sum_{k=1}^{K} \beta_{k}(E) .
$$

Construction of MP DCSs is somewhat generic in that one simply choses a preferred DCS representation using some number of parameters - in this case, discrete points and weights - and then sets up a system of equations for the parameters by enforcing moment preservation. The moment-preservation constraint is stated as follows:

$$
\Sigma_{a, \ell}=\tilde{\Sigma}_{a, \ell}, \quad \ell=1,2, \ldots, L,
$$

and

$$
\Sigma_{e, n}=\tilde{\Sigma}_{e, n}, \quad n=1,2, \ldots, N,
$$

for elastic Coulomb collisions and impact-ionization collisions respectively. By introducing Eqs. (12) and (13) into Eqs. (15) and (16) respectively, one can form a system of equations for the discrete points and weights. The resulting system of equations lends itself to a quadrature-based solution approach that is described in Dixon et al. ${ }^{(\mathbf{8})}$

As indicated in Eqs. (15) and (16), one enforces preservation of the higher-order moments and intentionally allows the zeroth moment to vary. In doing, the zeroth moment is self-determined by the DCS construction process and results in reduction of the zeroth moment (or the total cross section). This effect is seen clearly in Figs. (1) and (2) where the ratio of the approximate total cross section to the true total cross section is presented. Note that as the particle energy increases, the ratio grows because the DCS becomes more peaked at higher energies, thus, enhancing the impact of the MP DCS construction process. In contrast, increasing the number of points and weights reduces this effect because increasing the number of points and weights increases the level of moment preservation and the MP DCSs effectively go over to the analog DCSs.

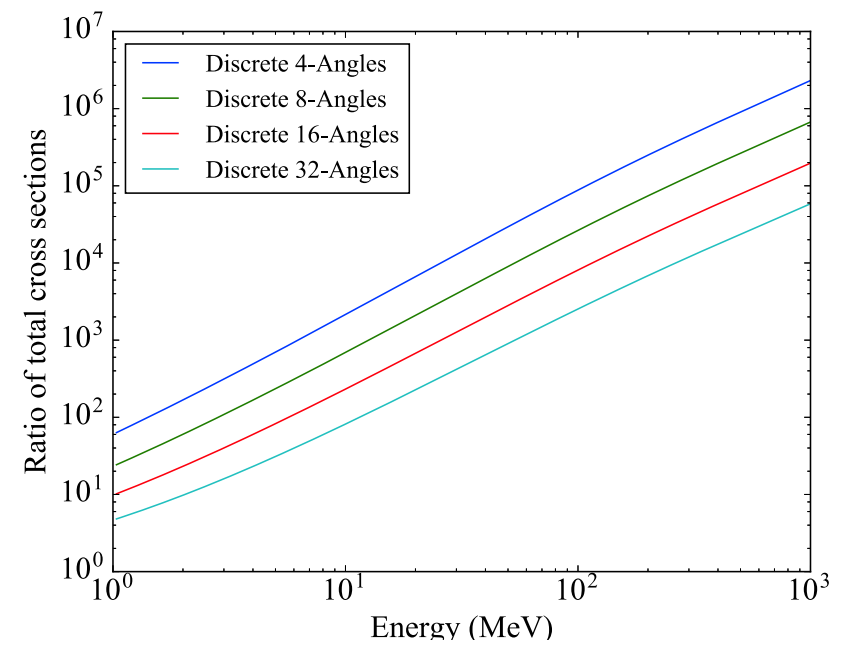

Figure 1: Comparison of analog and MP total cross sections for various discrete angular deflection models.

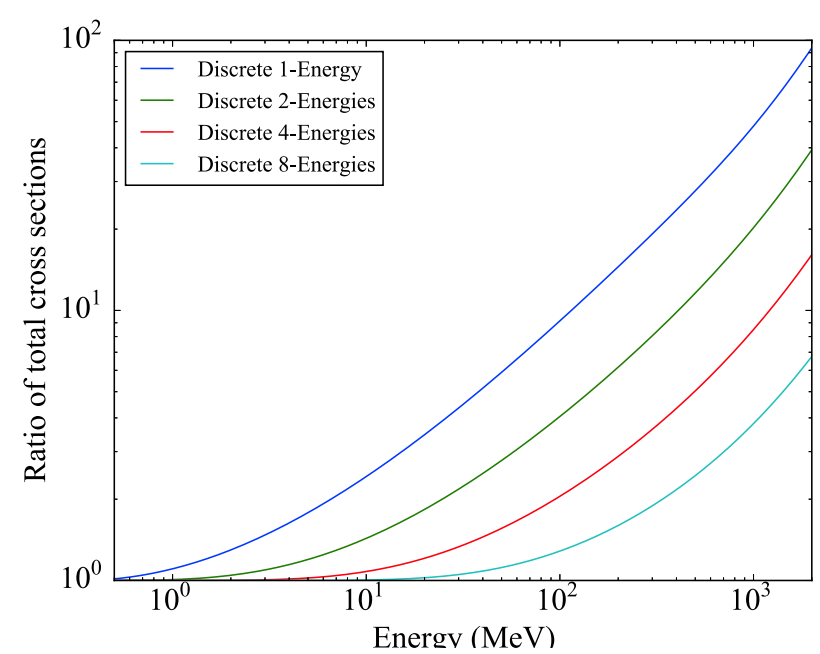

Figure 2: Comparison of analog and MP total cross sections for various discrete impact-ionization models.

\section{Results}

In the following sections, we present results from infinite medium transport models (angular distributions and so on), straight-ahead transport models (energy spectra), and a more complete transport model (energy deposition profiles).

\section{Angular, longitudinal, and lateral distributions}

Here, we study angular, longitudinal, and lateral distribution for protons (1-, 10-, and 100-MeV) in infinite media. Protons start at the origin and are followed until they reach a termination distance that is given by some fraction of the proton transport mean free path. Along the traversed path, protons may only undergo Coulombic collisions with target atoms and energy losses resulting during the collisions are neglected. When the proton reaches the end of the prescribed distance, a tally is made of the current direction, and the lateral and longitudinal displacement. Corresponding results are presented in Figs. 6 8 and Table 1.

In Fig. 6, angular distributions are presented for 1-, 10-, $100-\mathrm{MeV}$ protons traversing distances of $\Sigma_{t r} / 10$ and $\Sigma_{t r} / 3$ in copper. Numerical benchmarks are compared to either 4- or 8 -angle MP models. The angular distributions for $1-\mathrm{MeV}$ and $10-\mathrm{MeV}$ protons agree between the numerical benchmark and the MP models with exception of the 4-angle model for a traversed distance of $\Sigma_{t r} / 10$. At $100-\mathrm{MeV}$, we observe significant disagreement between the numerical benchmark and the MP models (this disagreement is reflected in the energy deposition results).

Longitudinal and lateral displacement distributions are presented in Figs. 7 and 8 (note the difference in scale for these results). Keeping in mind the difference in scale, Figs. 7 and 8 show stronger disagreement as a function of increasing energy; particularly, in the tails of the distribution. Once again, the 8-angle model was the only model tested with consistent agreement and this agreement is only observed at for $1-\mathrm{MeV}$ protons.

In general, the infinite medium results show that the discrete MP models tend to over-diffuse. This effect is exacerbated 
by increasing energy. Regarding efficiency, from Table 1 one might conclude that there is great potential for efficiency gains in the MP method. However, one will find that the MP method is very effective at extending the mean free path associated with Coulombic collisions with target atoms, but not as effective at extending the mean free path associated with impact-ionization. Therefore, in more realistic transport where MP models for Coulombic collisions with target atoms and electrons are combined, efficiency gains are limited by impact-ionization collisions, or the latter.

Table 1: Speed-up factors for computation of pathlength distributions in copper using 8-angle model. Speed-up factors are the ratio of the analog model simulation CPU time to that of a discrete model simulation CPU time .

\begin{tabular}{cccc}
\hline Pathlength $(\mathrm{cm})$ & $1-\mathrm{MeV}$ & $10-\mathrm{MeV}$ & $100-\mathrm{MeV}$ \\
\hline $0.1 \sigma_{t r}$ & 48 & 157 & 4033 \\
$0.3 \sigma_{t r}$ & 50 & 168 & 4445 \\
\hline
\end{tabular}

\section{Energy spectra}

In this section, we study energy spectra of protons emerging from tungsten slabs with increasing thicknesses in a straightahead transport model where protons are restricted to energyloss collisions. Fig. 9 presents a comparison of numerical benchmarks generated using the analog physics with various discrete models. For each of the spectra results, $10^{6}$ particles were followed. Results indicating levels of accuracy and efficiency are compared in Fig. 9 and in Table 1 respectively.

For thinner slabs, the distributions are skewed towards higher energies and preservation of additional moments is required to capture this structure. As the slab thickness increases, the spectra broadens, indicating that higher-order moments are less important and a model with two discrete energies (preserving four moments) is sufficient. These results illustrate an advantage and a limitation of the MP method. That is, as the slab thickness is reduced to $0.1-\mathrm{cm}$, we observe discrete artifacts when using eight discrete points and weights. The artifacts arise in problems where an insufficient number of collisions occur resulting in preferred channels of energy-loss. This is easily resolved by increasing the number of discrete points and weights, or using alternative representations such as the hybrid model. $^{(8)}$ The opposite effect is observed in the $20-\mathrm{cm}$ slab, where significant spreading in energy occurs via an increased number of collisions. Under these conditions, one can reduce the number of points and weights while maintaining agreement with the numerical benchmark. These two observables highlight one of the key features of the MP method - one can systematically control the accuracy of the method in varying scattering regimes. Table 1 shows that the efficiency of the MP method is model dependent and ranges from roughly a factor of 4 to 50 times faster than the computer time required to generate the numerical benchmark. This indicates that there is a clear connection between accuracy and efficiency in the MP method. As with most approximations, one must strike a balance between efficiency and accuracy.
Table 2: Speed-up factors for straight-ahead transport in tungsten. Speed-up factors are the ratio of the analog model simulation CPU time to that of a discrete model simulation CPU time.

Discrete Model

\begin{tabular}{ccccc} 
& & & & \\
\cline { 2 - 5 } Thickness $(\mathrm{cm})$ & 1-Energy & 2-Energies & 4-Energies & 8-Energies \\
\hline 0.1 & 28 & 14 & 8 & 4 \\
0.5 & 42 & 20 & 9 & 4 \\
1.0 & 54 & 27 & 9 & 5 \\
2.0 & 53 & 27 & 9 & 4 \\
5.0 & 47 & 22 & 10 & 4 \\
20.0 & 49 & 21 & 9 & 4 \\
\hline
\end{tabular}

\section{Energy deposition}

In this section, energy deposition profiles for 7-MeV and 235$\mathrm{MeV}$ protons in silicon and tungsten, respectively, are presented. In Figs. 3 - 5, energy deposition profiles for 7-MeV protons in silicon generated using various approximations are compared to a numerical benchmark. In Fig. 3 two different discrete MP models are compared to a numerical benchmark generated using the analog DCSs presented in Eqs. (1) and (3). As seen in the energy deposition profile, the two MP models (4-angles, 4-energies and 8-angles, 8-energies) are indistinguishable on visual inspection. However, the relative difference plot below the energy deposition profiles in Fig. 3 shows that there is disagreement in the first cell (closest to the source) and is between 3-4\%. Agreement improves to fractions of a percent in the following cells until nearing the region of peak dose, where disagreement increases to a maximum of 2-3\%. Finally, disagreement in the tail of the profile, or beyond the peak dose region, grows substantially. However, in this region, the energy deposited is rapidly falling to zero and uncertainty tends to overwhelm the solution.

In Fig. 4, three different approximations are compared to a numerical benchmark, where angular deflection is given by the analog DCS in Eq. (1) and energy-loss is given by the G4hIonisation model ${ }^{(\mathbf{1 1})}$ (a multiple energy-loss straggling model). Similarly, the approximate models all combine the G4hIonisation model with various approximation representations of angular deflection (discrete MP representations or the Geant4 proton multiple-scattering model - i.e., Geant4 Protons). In contrast to the results in Fig. 3, there is no significant disagreement in the first cell. Like the results in Fig. 3, observable disagreement occurs in regions nearby the peak deposition. The Geant4 proton model tends to underestimate more significantly in the approach to the peak, while the 4-angle discrete MP model shows the greatest disagreement in the peak deposition region. In all cases, the greatest disagreement is with $4 \%$ of the numerical benchmark.

In Fig. 5, energy deposition profiles for 235-MeV protons in tungsten generated using various approximations are compared to a numerical benchmark. Again, the numerical benchmark was generated using the analog DCS in Eq. (1) for angular deflection and the G4hIonisation model for energy-loss. Two approximations are compared - an 8-angle discrete MP model and the Geant4 multiple-scatter model (each model was combined with the G4hIonisation model). Figure 5 shows clear 
disagreement between the numerical benchmark and the two approximations, where the most significant disagreement is attributed to the MP model. This disagreement is supported by the observations made in the discussion of the angular, longitudinal, and lateral distribution section, where it was noted that the discrete MP models tended to over-diffuse the solution - particularly, as energy increases.
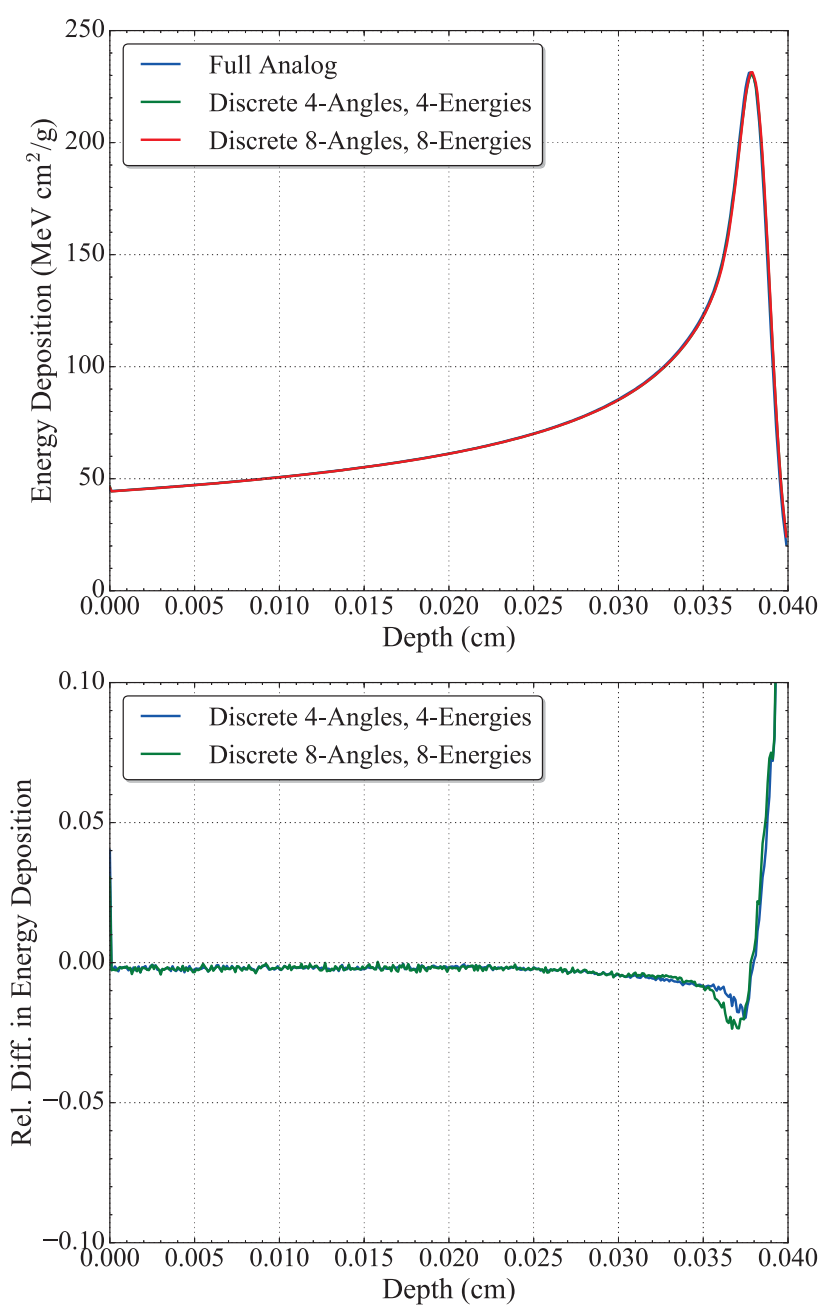

Figure 3: Comparison of proton energy deposition profiles for 7MeV protons incident on a 1-D silicon slab. The benchmark was generated using the analog cross sections described in section 2, while the other profiles were generated using MP models. Relative differences are presented below the energy deposition profiles where differences are measured with respect to the benchmark.

In all cases, efficiency was strongly dependent on the range cut applied to electrons. That is, when applying a large range cut to electrons, the distance to a hard collision grows significantly. In effect, the G4hIonisation model is effectively an application of CSDA tables along the path of the proton between angular deflection collisions. Under these conditions, efficiency gains - measured with respect to the time required to compute the numerical benchmark - were on the order of $10^{2}$. However, as the range cut is reduced, efficiency gains are reduced to less than a factor of ten or the time to completion using the approximate model is less than 10 times that of the analog model. From this, one can conclude that efficiency is dominated by impact-ionization collisions. In other words, one can develop models that are highly effective at extending the mean free path associated with angular deflection, but the effectiveness of the aforementioned model is limited by the shortest mean free path across all models considered.
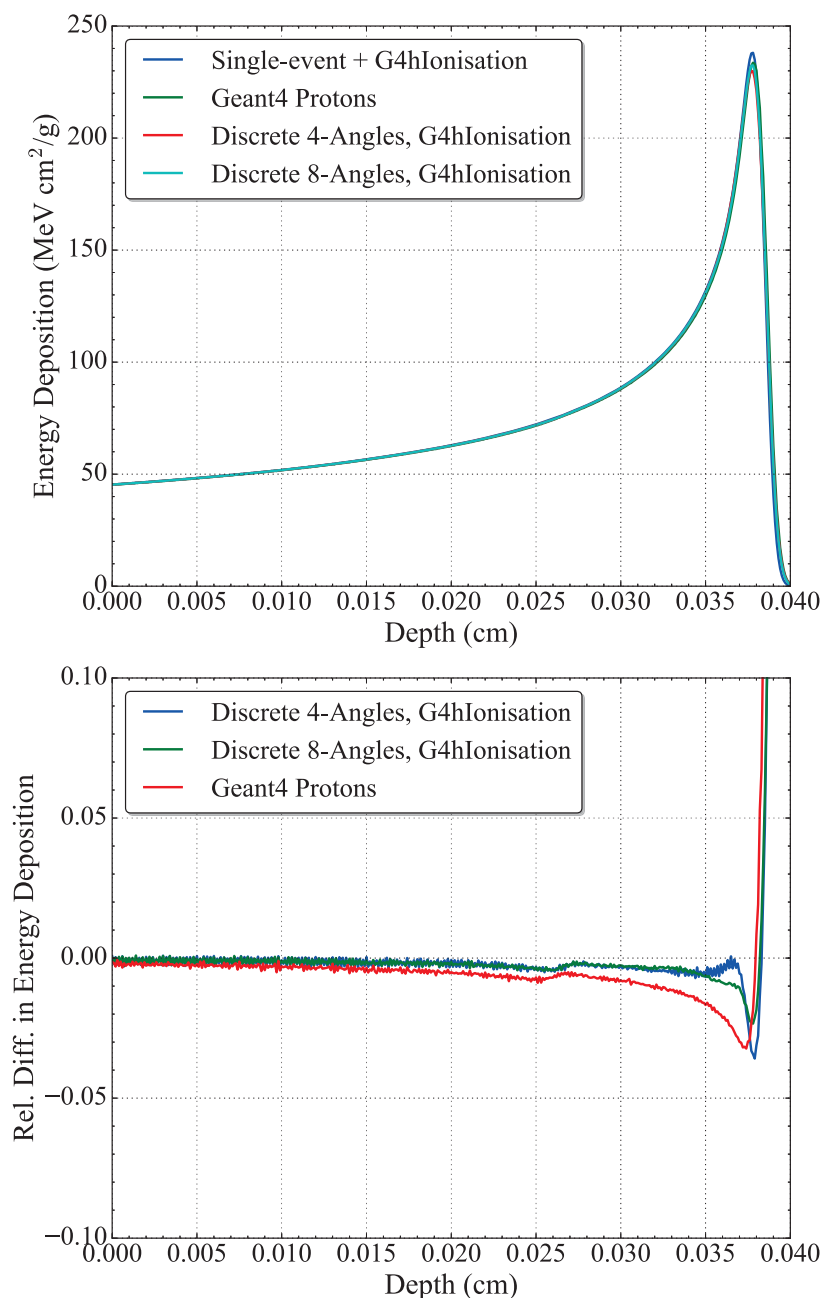

Figure 4: Comparison of proton energy deposition profiles for 7-MeV protons incident on a 1-D silicon slab. The numerical benchmark was generated using an analog Coulomb scattering model combined with the G4hIonization model (blue). The other profiles were generated using approximate methods including the standard Geant 4 multiple-scattering treatment for protons (green), the discrete 4-angle MP model combined with the G4hIonization model (red), and the discrete 8-angle MP model combined with the G4hIonization model (teal). In addition, relative differences are presented below the energy deposition profiles where differences are measured with respect to the numerical benchmark.

\section{Conclusions}

This paper served as an initial test of the applicability of the MP method for proton transport. A variety of discrete MP models were tested for use in computing angular, lateral, and longitudinal distributions, energy spectra, and energy deposition profiles. Consistent with previous work on electrons, accuracy and efficiency are both model and problem dependent. That is, efficient, low-order models can be used in thick problems where signif- 


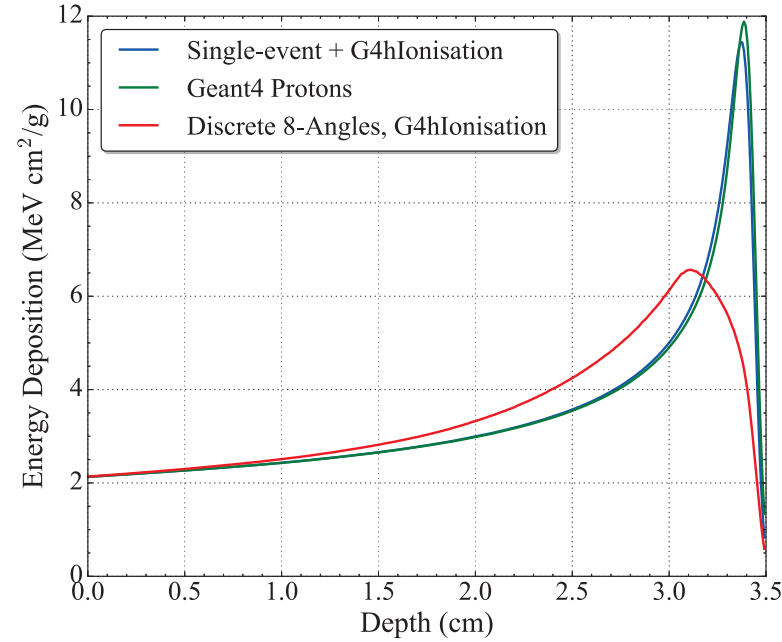

Figure 5: Comparison of proton energy deposition profiles for 235-MeV protons incident on a 1-D tungsten slab. The numerical benchmark was generated using an analog Coulomb scattering model combined with the G4hIonization model (blue). The other profiles were generated using approximate methods including the standard Geant 4 multiple-scattering treatment for protons (green) and the discrete 8-angle MP model combined with the G4hIonization model (red). Relative differences are not presented for this case because the disagreement between the MP model and the numerical benchmark is .

icant spreading of the beam occurs. Alternatively, in peaked regimes, such as very thin slabs, less-efficient, higher-order models can be applied.

While there were similarities between observations made in this work and in previous work on electrons, systematic accuracy was not not observed where discrete representations of angular deflection were applied. In such problems, disagreement worsened with increasing energy. A somewhat speculative conclusion is that the discrete representation angular deflection is not appropriate for protons given the extreme peakedness considerably more so than for electrons - of the analog DCS. Alternatively, it is possible that there is an inconsistency in the generation and the application of the discrete DCS for angular deflection, particularly, when boosting the deflection angle sampled in the COM to the lab frame. Moreover, it is possible that the expression for the accompanying energy-loss derived from relativistic kinematics should be considered when computing the discrete points and weights. That is, one might use Legendre/energy-loss moments with cross terms as input for the discrete cross section generation tool. Assuming that the discrete models are inappropriate for angular deflection, there are alternative MP formulations. Of particular interest is the hybrid model, ${ }^{(\mathbf{8})}$ where discrete points and weights are applied over the small range corresponding to the peak of the DCS and the tail is captured exactly by the analog DCS.

Regarding the discrete impact-ionization models, we observed satisfactory efficiency and accuracy. That is, in the straight-ahead problems, application of various discrete impactionization models showed good agreement with the numerical benchmarks and efficiency gains ranged from 4 - 50 times faster than the analog model.

Finally, the Geant4 process and model classes used for elec- trons were easily extended for use in treating protons. This is because the process and model classes do not require modification, rather we simply supplied the new proton MP DCSs. So, generalization of the MP method to protons and heavy ions will mostly require identifying ideal analog DCSs and generating the corresponding MP DCSs that are appropriate for these particle types.

\section{Acknowledgments}

This work was funded by the US Department of Energy's National Nuclear Security Administration, under Los Alamos National Security, LLC, Contract No. DE-AC52-06NA25396.

\section{References}

1) R. Evans, The Atomic Nucleus, McGraw-Hill, New York, tenth printing edition, (1955).

2) M. Berger, "Monte Carlo Calculation of the Penetration and Diffusion of Fast Charged Particles," Proc. Methods in Computational Physics, volume 1 of Statistical Physics, p. 135-215, Advances in Research and Applications, Academic Press, 1963.

3) A. Bielajew and D. D. O. Rogers, "PRESTA: The Parameter Reduced Electron-Step Transport Algorithm for Electron Monte Carlo Transport," Nucl. Instr. Meth. B, 18, 165-181 (1986).

4) A. Bielajew and F. Salvat, "Improved electron transport mechanics in the PENELOPE Monte-Carlo model," Nucl. Instr. Meth. B, 173, 332-343 (2001).

5) F. Salvat, J. M. Fernández-Varea, and J. Sempau, PENELOPE2011: A Code System for Monte Carlo Simulation of Electron and Photon Transport, Universitat de Barcelona, 2011.

6) G. Molière, "Theori der Streuung schneller geladener Teilchen I: Einzelstreuung am abgeschirmten Coulomb-Field," Z. Naturforsch., 2a, 133 (1947).

7) P. V. Vavilov, "Ionization Losses of High-Energy Heavy Particles," Sov. Phys. JETP, 5, 4, 920-923 (1957).

8) D. Dixon, A. Prinja, and B. Franke, "A computationally efficient moment-preserving Monte Carlo electron transport method with implementation in Geant4," Nucl. Instr. Meth. B, 359, 20-35 (2015).

9) M. J. Boschini et al., "Nuclear and Non-Ionizing Energy-Loss for Coulomb Scattered Particles from Low Energy up to Relativistic Regime in Space Nuclear and Non-Ionizing Energy-Loss for Coulomb Scattered Particles from Low Energy up to Relativistic Regime in Space Radiation Environment," Proceedings of the ICATPP Conference on Cosmic Rays for Particle and Astroparticle Physics (2010).

10) Principles of Radiation Interaction in Matter and Detection, World Scientific, $4^{\text {th }}$ edition, (2016).

11) S. Agostinelli et al., "Geant4-a simulation toolkit," Nucl. Instr. Meth. A, 506, 250-303 (2003). 

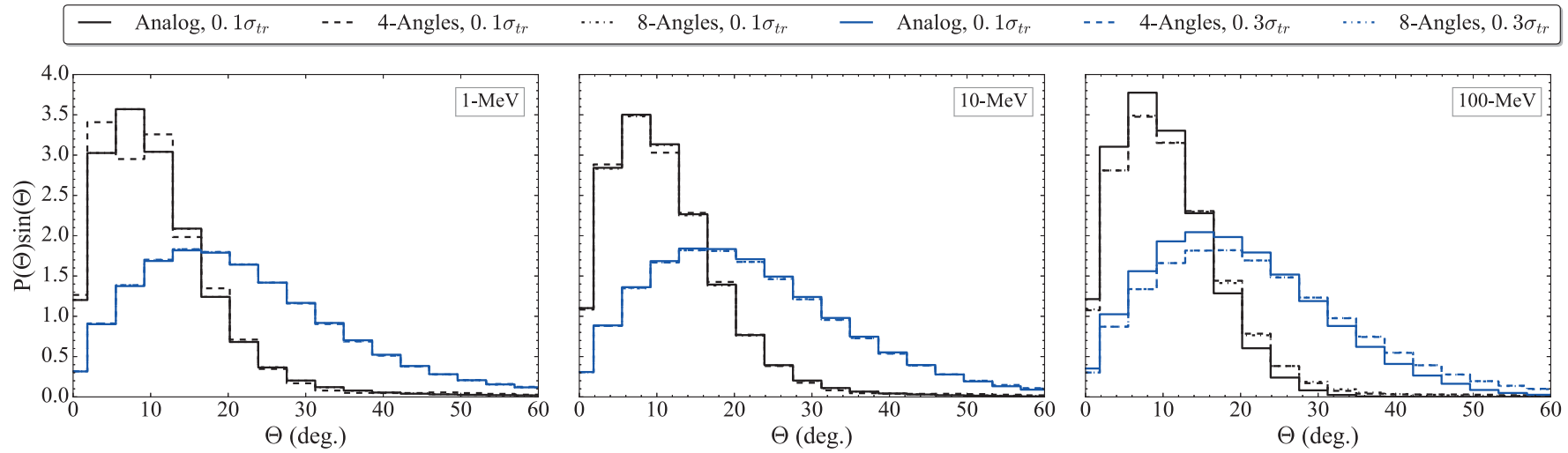

Figure 6: Comparison of angular distributions for $1-\mathrm{MeV}, 10-\mathrm{MeV}$, and $100-\mathrm{MeV}$ protons in an infinite medium of copper after traveling a distance of $\Sigma_{t r} / 10$ and $\Sigma_{t r} / 3$. The analog solutions (solid curve) were obtained using the Wentzel DCS and compared to a 4-angle (dash curve) and 8-angle (dash-dot curve) discrete model.
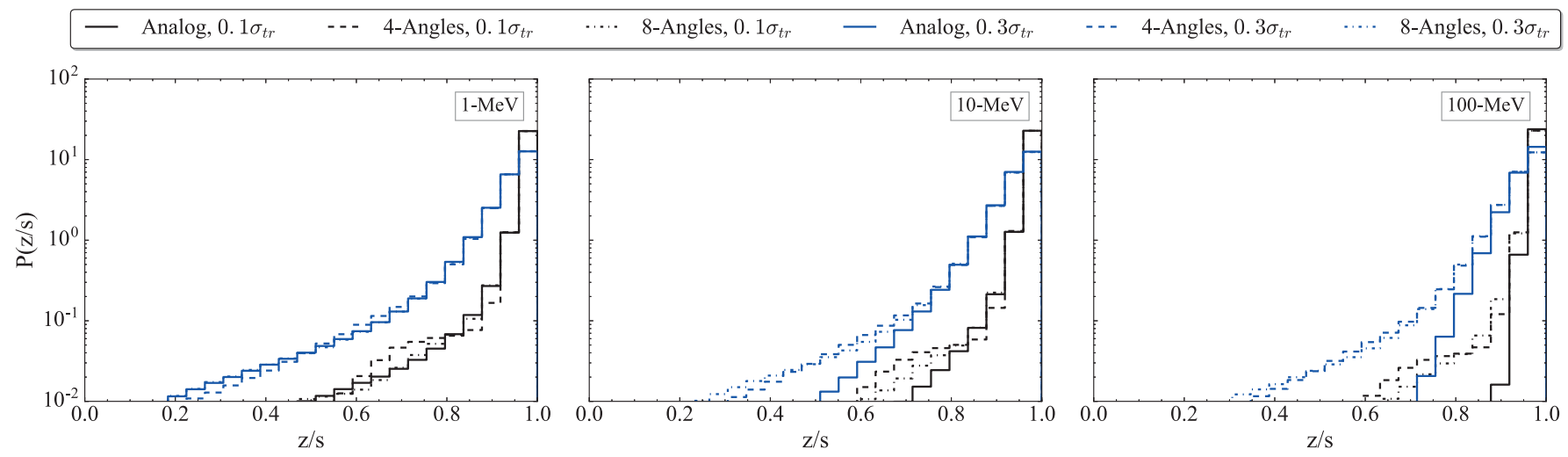

Figure 7: Comparison of longitudinal distributions for $1-\mathrm{MeV}, 10-\mathrm{MeV}$, and $100-\mathrm{MeV}$ protons in an infinite medium of copper after traveling a distance of $\Sigma_{t r} / 10$ and $\Sigma_{t r} / 3$. The analog solutions (solid curve) were obtained using the Wentzel DCS and compared to a 4-angle (dash curve) and 8-angle (dash-dot curve) discrete model.
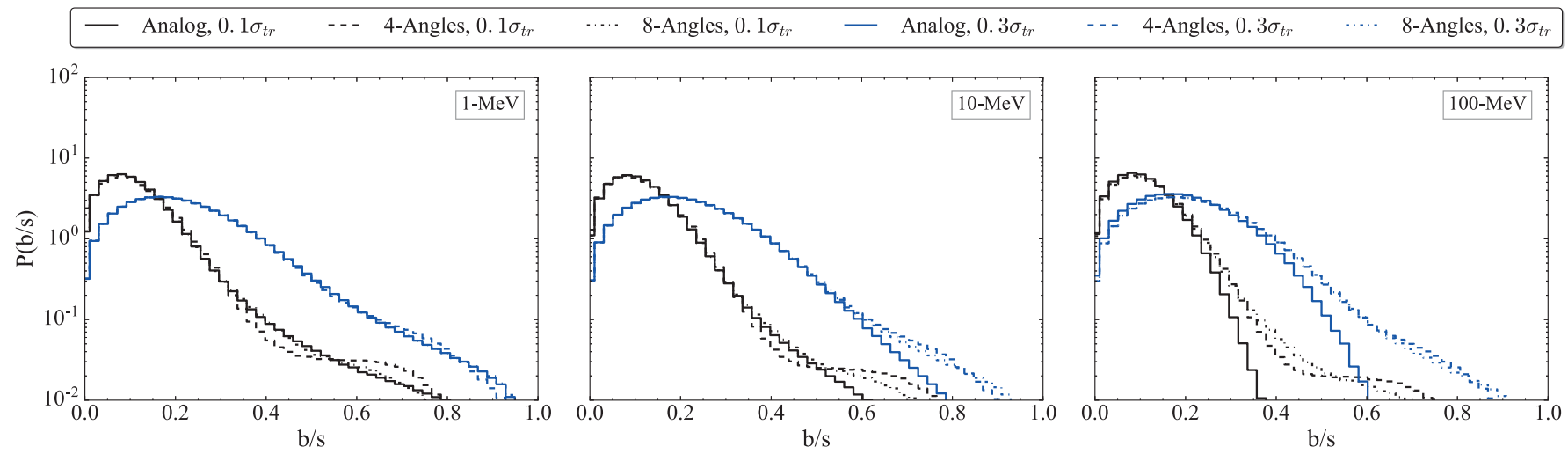

Figure 8: Comparison of lateral distributions for $1-\mathrm{MeV}, 10-\mathrm{MeV}$, and $100-\mathrm{MeV}$ protons in an infinite medium of copper after traveling a distance of $\Sigma_{t r} / 10$ and $\Sigma_{t r} / 3$. The analog solutions (solid curve) were obtained using the Wentzel DCS and compared to a 4-angle (dash curve) and 8-angle (dash-dot curve) discrete model. 


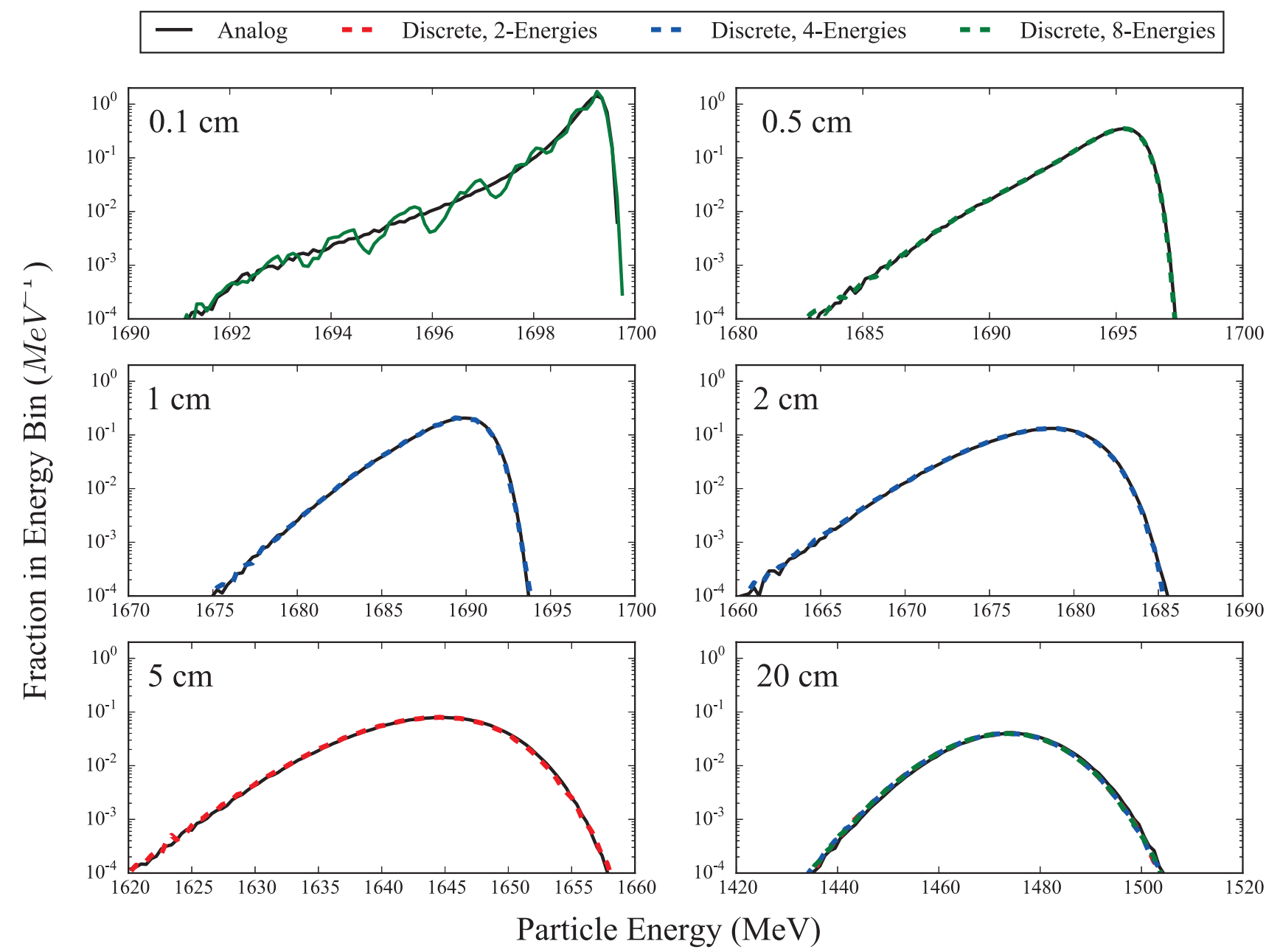

Figure 9: Comparison of energy spectra in 0.1-, 0.5-, 1-, 2-, 5-, and 20-cm tungsten slabs calculated using the analog model and various discrete models. In thicker slabs (bottom row), low-order models are sufficient, while in very thin (top row) slabs higherorder models are required. For the extreme case $(0.1-\mathrm{cm})$ discrete artifacts are present with the most accurate model tested (eight discrete energies). 